\title{
Proposta de um fluxograma para o processo de desenvolvimento de produtos de Tecnologia Assistiva
}

\author{
Fernanda do Nascimento Maia ${ }^{a}$, Sydney Fernandes de Freitas ${ }^{\mathrm{b}}$ \\ anstituto Fernandes Figueira - IFF, Fundação Oswaldo Cruz - FIOCRUZ, Rio de Janeiro, RJ, Brasil

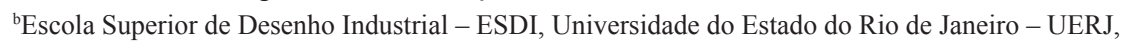 \\ Rio de Janeiro, RJ, Brasil
}

\begin{abstract}
Resumo: Em entrevistas exploratórias e observações assistemáticas com profissionais atuantes no campo de reabilitação de pessoas com deficiência observou-se falta de sistematização no processo de desenvolvimento de recursos de tecnologia assistiva, resultando em produtos com baixa qualidade de usabilidade. Esta pesquisa teve como objetivo principal contribuir, através de uma proposta metodológica, para a formação do terapeuta ocupacional no processo de desenvolvimento de objetos para pessoas com deficiência. Realizou levantamento bibliográfico na área de produtos para pessoas com deficiência; apresentação da base teórica relativa ao design de produtos; entrevistas semiestruturadas com terapeutas ocupacionais que produzem objetos de Tecnologia Assistiva; entrevistas semiestruturadas com terapeutas ocupacionais que realizaram encaminhamento de confecção de produto de Tecnologia Assistiva a outro profissional; avaliação dos aspectos de usabilidade de produtos de Tecnologia Assistiva confeccionados por terapeutas ocupacionais; levantamento das disciplinas relacionadas com Tecnologia Assistiva ministradas nos cursos de Terapia Ocupacional do Rio de Janeiro e pesquisa através de questionário com professores das referidas disciplinas; análise crítica e um paralelo entre a proposta de Ergodesign de Frisoni (2000) e as etapas e fases mencionadas pelos terapeutas ocupacionais na pesquisa de campo. Concluímos com a proposição de um fluxograma para o desenvolvimento de recursos de Tecnologia Assistiva.
\end{abstract}

Palavras-chave: Equipamentos de Autoajuda, Pessoas com Deficiência, Métodos, Tecnologia de Produtos.

\section{Proposal of a flowchart for the development process of Assistive Technology products}

\begin{abstract}
In exploratory interviews and unsystematic observations with professionals working in the field of rehabilitation of persons with disabilities, we observed that there is a lack of systematic development in the process of assistive technology resources, resulting in products with low quality of usability. The main objective of the present study was to contribute, through a methodological proposal, to the formation of occupational therapists in the process of developing objects for persons with disabilities. The methodology used was a brief review of the steps taken in this research. The survey was conducted and recorded relying on the following steps: literature review; organization of the research project; presentation of data from field research conducted with occupational therapists who produce objects of assistive technology; assessment and interview with users; application of questionnaires to teachers of assistive technology disciplines; interviews with occupational therapists who perform making assistive technology products to other professionals; proposal of a flowchart for the development of assistive technology resources.
\end{abstract}

Keywords: Self-help Equipment, Persons with Disabilities, Methods, Product Technology.

Autor para correspondência: Fernanda do Nascimento Maia, Instituto Fernandes Figueira, Setor - Programa Saúde e Brincar, Fundação Oswaldo Cruz, Av. Rui Barbosa, 716, Flamengo, CEP 22250-020, Rio de Janeiro, RJ, Brasil, e-mail: fernanda.maia@iff.fiocruz.br

Recebido em 1/11/2013; Revisão em 20/5/2014; Aceito em 20/6/2014. 


\section{Introdução}

No Brasil, poucos são os produtos acessíveis ou especificamente projetados para pessoas com deficiência ou idosos. A maioria dos produtos utilizados por pessoas com déficits funcionais é importada ou confeccionada por profissionais sem formação específica em atividades projetuais, como é o caso dos terapeutas ocupacionais. A tradição acrítica de se desenvolver produtos assistematicamente contribui para a estagnação do desenvolvimento tecnológico dos terapeutas ocupacionais. Diferentemente do que já acontece em países com uma maior produçấo tecnológica voltada para as pessoas com limitaçóes funcionais, os terapeutas ocupacionais no Brasil continuam apenas adaptando objetos existentes no mercado ou repetindo modelos de desenvolvimento de recursos de Tecnologia Assistiva (TA) incompletos, o que atrapalha a produção de recursos realmente adequados ao usuário. A carência de pesquisas, de produtos nacionais voltados para essa clientela e a dificuldade de importação de tais produtos leva à manutençáo desse quadro. Dentro dessa realidade a pesquisa teve como objetivo contribuir, através de uma proposta metodológica, para a formaçâo do terapeuta ocupacional no processo de desenvolvimento de objetos para pessoas com deficiência.

\section{Métodos e técnicas de desenvolvimento de Tecnologia Assistiva}

Metodologia é a área do saber que estuda o método. Segundo Thiollent (apud Frisoni, 2000), a metodologia é uma disciplina cujo objetivo consiste em: analisar as características dos vários métodos disponíveis; avaliar as capacidades, potencialidades, limitaçôes e distorçôes desses métodos; e criticar os pressupostos ou as implicações da utilizaçáo desses métodos.

Por método podemos entender um conjunto de procedimentos racionais, explícitos e sistemáticos, postos em prática para se alcançar resultados teóricos ou concretos ditos verdadeiros, de acordo com algum critério estabelecido (CIPINIUK; PORTINARI, 2006).

Ser criativo implica ser disciplinado e persistente. Ser organizado facilita o raciocínio, poupa tempo e diminui os riscos. Faz sobrar mais tempo para pensar em soluçóes e apurar as ideias. A desordem diminui a possibilidade de criação. Desarrumar é criar obstáculos desnecessários, é consumir o oxigênio do ambiente de projeto, é sufocar a imaginação. $\mathrm{O}$ mecanismo da desordem (na realidade um antimecanismo) é "espaçoso", porque consome espaço vital, e é contaminante, porque se propaga e imobiliza a criação (LARICA, 2006, p. 93).

Dessa forma podemos pensar o método como uma forma de organizar e potencializar o processo criativo.

No âmbito internacional encontramos diversas pesquisas relacionadas às metodologias de desenvolvimento de TA e a fatores diretamente a elas relacionados. Alguns estudos relatam um problema de abandono dos recursos de TA (PHILLIPS; ZHAO, 1993; HOCKING, 1999; SCHERER, 2002; RIEMER-REISS; WACKER, 2000; SCHERER; CRADDOCK, 2002). Outros estudos são direcionados a questôes psicossociais, usabilidade e qualidade de vida relacionadas à TA (DAY; JUTAI, 1996; JUTAI, 1999; WEISSLAMBROU et al., 2000; LUPTON; SEYMOUR, 2000; SCHERER, 2002; LOUISE-BENDER; KIM; WEINER, 2002; DESMET; DIJKHUIS, 2003; SCHERER et al., 2005).

Ainda no âmbito internacional, tendo como base o Design Centrado no Usuário, algumas metodologias de desenvolvimento de produtos de TA vêm sendo discutidas nos últimos anos. Em 1996, foi lançado o Userfit, um guia dividido em várias partes que tem como proposta ser um manual prático para o design centrado no usuário para TA (POULSON; ASHBY; RICHARDSON, 1996). Em 2009, Wu, Ma e Chang propõem o Assistive Design Analysis Method for Objective User Criteria (AD-SWOT) e o Assistive Device Design Development Method (AD-TOWS), propostas que podem ser consideradas protocolos de avaliaçáo do usuário (com grande foco nas questóes motoras) e de seleção de alternativas.

No Brasil ainda são poucos os trabalhos nessa área, porém com um crescente aumento nos últimos anos. Ao pesquisarmos trabalhos na área de desenvolvimento de Tecnologia Assistiva encontramos principalmente relatos de experiências e propostas de soluções ou produtos específicos. Nesse caso são maiores os resultados nas áreas de Tecnologia de Informação e Educação Especial.

Mais especificamente em metodologia para o desenvolvimento ou prescrição de recursos de TA encontramos alguns trabalhos que discutem a necessidade de se pensar essas metodologias, como os de Mallin (2004), Souza (2004), Bersch (2009) e Rocha (2010). Pensando na necessidade de termos 
modelos voltados para a realidade brasileira, a Secretaria de Educação Especial publicou em 2002 um guia denominado Recursos Pedagógicos Adaptados, onde Manzini e Santos (2002) descrevem a proposta de um processo para o desenvolvimento de ajudas técnicas (Tecnologia Assistiva). Mais recentemente, Alves (2013) realizou uma análise e adaptação transcultural do modelo conceitual Matching person and Technology e da avaliação Assistive Technology Device Predisposition Assessment, buscando oferecer uma forma sistematizada de indicaçáo de recursos de TA pertinente à realidade nacional.

Nas questôes relativas ao abandono dos recursos de TA, não existem estudos sistematizados realizados no território brasileiro, porém é possível observarmos empiricamente, a partir do relato da experiência de profissionais de saúde, que essa problemática também se apresenta.

\section{Etapas metodológicas da pesquisa}

O presente artigo visa apresentar de forma sucinta as etapas percorridas pela pesquisa de mestrado que resultou na dissertação intitulada "A contribuição da Metodologia de Projeto em Design no processo de desenvolvimento de recursos de Tecnologia Assistiva”. A mesma foi realizada e registrada com as seguintes etapas:

- Levantamento bibliográfico. Apresentação das teorias e conceitos relevantes na área de produtos para pessoas com deficiência. Discussão dos modelos de ação na área de reabilitação (modelo médico/modelo social), dos princípios que norteiam o desenvolvimento de produtos para pessoas com deficiência (Design Universal/ Tecnologia Assistiva/Design Inclusivo), do mercado brasileiro desses produtos, do público alvo e da Terapia Ocupacional como designer de produtos dentro do contexto da TA.

- Levantamento bibliográfico. Apresentação da base teórica relativa ao design de produtos. Discussão da importância do uso de uma metodologia no processo de criação. Apresentação da pesquisa de Frisoni (2000) sobre metodologias na área de design de produto e ergonomia; dos aspectos da relação do usuário com o objeto que são relevantes tanto na visão da ergonomia quanto do design; da proposta de Frisoni (2000) de uma metodologia de Ergodesign.
- Métodos e procedimentos utilizados na pesquisa de campo: entrevistas semiestruturadas com terapeutas ocupacionais que produzem objetos de TA; entrevistas semiestruturadas com terapeutas ocupacionais que realizaram encaminhamento de confecção de produto de TA a outro profissional; avaliação dos aspectos de usabilidade de produtos de TA confeccionados por terapeutas ocupacionais; levantamento das disciplinas relacionadas com TA ministradas nos cursos de Terapia Ocupacional (TO) do Rio de Janeiro e pesquisa através de questionário com professores das referidas disciplinas.

- Apresentaçáo dos dados da pesquisa de campo realizada. Apresentação de uma análise crítica e um paralelo entre a proposta de Ergodesign de Frisoni (2000) e as etapas e fases mencionadas pelos terapeutas ocupacionais na pesquisa de campo. Proposta de uma metodologia com um conjunto de técnicas que contribuam para o desenvolvimento mais eficaz, eficiente e satisfatório de produto para indivíduos com deficiência, por terapeutas ocupacionais.

\section{Objetivos}

A pesquisa teve como objetivo principal contribuir, através de uma proposta metodológica, para a formação do terapeuta ocupacional no processo de desenvolvimento de objetos para pessoas com deficiência.

As pesquisas de campo tiveram como objetivo: entender a metodologia utilizada pelos terapeutas ocupacionais no desenvolvimento de produtos de TA e os conceitos por eles considerados relevantes nesse processo; identificar como se dá o processo de encaminhamento de confecção para outro profissional; identificar as metodologias ensinadas pelos professores das disciplinas de TA no estado do Rio de Janeiro e a relação do usuário com os objetos produzidos pelos terapeutas ocupacionais.

\section{Resultados}

Ao longo da pesquisa foi possível observar a necessidade de um processo sistematizado para o desenvolvimento de produtos de TA. No resultado da pesquisa de campo destacaram-se os seguintes aspectos: falta de um método por parte dos terapeutas ocupacionais no desenvolvimento de objetos de TA; a não consideração como importante, por 
parte desses profissionais, de fatores como cor do objeto, idade do usuário e estética do produto, em contraponto com os usuários, que apresentam fatores relacionados à usabilidade e estética como relevantes; não reconhecimento, por parte dos profissionais, dos familiares como usuários secundários; o empirismo no processo de avaliaçáo do produto por parte dos terapeutas que encaminham o produto para confecção por outro profissional; a não menção de tópicos relativos ao método para o desenvolvimento de TA nas disciplinas de TA dos cursos de graduaçáo de TO do Rio de Janeiro.

\section{Método para o desenvolvimento de recursos de Tecnologia Assistiva baseado no Ergodesign}

Com base nas informação da pesquisa de campo foi realizado um paralelo entre a proposta de Ergodesign de Frisoni (2000) e as etapas e questóes relevantes ao processo verificadas a partir da pesquisa de campo.

A escolha da pesquisa citada se deu pelo fato de a mesma englobar diversas teorias nas áreas de design $\mathrm{e}$ da ergonomia, propondo assim um fluxograma amplo para o desenvolvimento de produtos que atendessem às necessidades do mercado e, principalmente, do usuário.

A partir dos resultados referentes às respostas dos terapeutas ocupacionais sobre como eles desenvolviam os recursos de TA, foi desenvolvido um quadro demonstrativo com as etapas e fases por eles mencionadas, como apresentado na Figura 1.

Foram feitas modificações no fluxograma buscando refletir as necessidades dos terapeutas ocupacionais e usuários no processo de desenvolvimento de produtos de TA utilizando, dentro do possível, a nomenclatura mencionada pelos terapeutas ocupacionais. Após as modificaçóes, foi proposto o fluxograma apresentado nas Figuras 2 e 3, para o desenvolvimento de produtos de TA.

\begin{tabular}{|c|c|}
\hline ETAPA & FASE \\
\hline \multicolumn{2}{|c|}{ - Definição do público-alvo $(10 \%)$} \\
\hline • Avaliação (100\%) & $\begin{array}{l}\text { - Física }(70 \%) \\
\text { - Observação da necessidade }(50 \%) \\
\text { - Verificação dos desejos }(50 \%) \\
\text { - Informações sobre ambiente }(30 \%) \\
\text { - Avaliação cognitiva }(30 \%) \\
\text { - Funcional }(30 \%) \\
\text { - Social }(10 \%) \\
\text { - Familiar }(10 \%) \\
\text { - Sensorial }(10 \%) \\
\text { - Recursos financeiros disponíveis }(10 \%) \\
\text { - Levantamento das expectativas }(10 \%) \\
\text { - Potenciais do usuário }(20 \%) \\
\text { - Levantamento dos dados antropométricos }(10 \%)\end{array}$ \\
\hline \multicolumn{2}{|c|}{ - Análise dos dados da avaliação (10\%) } \\
\hline \multicolumn{2}{|l|}{$\begin{array}{l}\text { - Levantamento das possíveis } \\
\text { soluçôes }(20 \%)\end{array}$} \\
\hline \multicolumn{2}{|l|}{ • Geração de ideias (10\%) } \\
\hline \multicolumn{2}{|c|}{ - Seleção entre as hipóteses $(10 \%)$} \\
\hline \multicolumn{2}{|l|}{ - Definição do produto $(10 \%)$} \\
\hline \multicolumn{2}{|l|}{ - Seleção do material (20\%) } \\
\hline \multicolumn{2}{|l|}{ - Planejamento $(20 \%)$} \\
\hline \multicolumn{2}{|l|}{ - Confecção do molde (20\%) } \\
\hline \multicolumn{2}{|l|}{ - Modelagem (10\%) } \\
\hline \multicolumn{2}{|c|}{ - Confecção do produto em si (100\%) } \\
\hline - Teste $(20 \%)$ & $\begin{array}{l}\text { - Se está correto funcionalmente }(10 \%) \\
\text { - Se o usuário gostou }(10 \%) \\
\text { - Se está confortável }(10 \%) \\
\text { - Se a cor está boa }(10 \%)\end{array}$ \\
\hline \multicolumn{2}{|l|}{ - Treinamento $(20 \%)$} \\
\hline \multicolumn{2}{|l|}{ - Uso $(50 \%)$} \\
\hline - Acompanhamento (40\%) & $\begin{array}{l}\text { - Reavaliação (10\%) } \\
\text { - Reavaliação em } 3 \text { meses }(10 \%) \\
\text { - Reavaliação em } 6 \text { meses }(10 \%) \\
\text { - Retirada ou não do recurso ou } \\
\text { remodelagem (10\%) }\end{array}$ \\
\hline
\end{tabular}

Figura 1. Etapas e fases mencionadas. 


\begin{tabular}{|c|c|c|c|c|}
\hline $\begin{array}{l}\text { Etapa 1: } \\
\text { Problematização } \\
\text { (parte 1) }\end{array}$ & $\begin{array}{l}\text { Etapa 1: } \\
\text { Problematização } \\
\text { (parte 2) }\end{array}$ & $\begin{array}{c}\text { Etapa 2: } \\
\text { Levantamento } \\
\text { de dados }\end{array}$ & $\begin{array}{c}\text { Etapa 3: } \\
\text { Análise } \\
\text { dos dados }\end{array}$ & $\begin{array}{c}\text { Etapa 4: } \\
\text { Projetação } \\
\text { do produto } \\
\end{array}$ \\
\hline \multirow{2}{*}{$\begin{array}{c}\text { Fase 1: } \\
\text { Definição do } \\
\text { publico alvo }\end{array}$} & \multirow{2}{*}{$\begin{array}{c}\text { Fase 8: } \\
\text { Observação das } \\
\text { necessidades }\end{array}$} & \multirow{15}{*}{\begin{tabular}{|c|} 
Fase 1: \\
Avaliação de \\
produtos \\
existentes \\
\end{tabular}} & & $\begin{array}{c}\text { Fase 1: } \\
\text { Planejamento }\end{array}$ \\
\hline & & & & \multirow{2}{*}{$\begin{array}{c}\text { Fase 2: } \\
\text { Modelagem } \\
\text { verbal }\end{array}$} \\
\hline Fase 2: & \multirow{2}{*}{$\begin{array}{c}\text { Fase 9: } \\
\text { Verificação } \\
\text { dos desejos }\end{array}$} & & & \\
\hline $\begin{array}{l}\text { Recursos } \\
\text { financeiros } \\
\text { disponíveis }\end{array}$ & & & & \multirow{2}{*}{$\begin{array}{c}\text { Fase 3: } \\
\text { Alocação } \\
\text { das funções }\end{array}$} \\
\hline & \multirow{2}{*}{$\begin{array}{l}\text { Fase 10: } \\
\text { Análise } \\
\text { Macro }\end{array}$} & & & \\
\hline $\begin{array}{c}\text { Fase 3: } \\
\text { Detalhamento }\end{array}$ & & & & \multirow{4}{*}{\begin{tabular}{|c|} 
Fase 4: \\
Ideação/seleção \\
da configuração \\
do sist. H-m/ \\
Possibilidades \\
da relação do \\
sujeito com o \\
produto \\
\end{tabular}} \\
\hline do problema & \multirow{2}{*}{$\begin{array}{l}\text { Fase 11: } \\
\text { Avaliação } \\
\text { da tarefa }\end{array}$} & & & \\
\hline $\begin{array}{c}\text { Fase 4: } \\
\text { Avaliacão }\end{array}$ & & & & \\
\hline física & \multirow{3}{*}{$\begin{array}{c}\text { Fase 12: } \\
\text { Análise } \\
\text { operacional }\end{array}$} & & & \\
\hline $\begin{array}{c}\text { Fase 5: } \\
\text { Avaliação }\end{array}$ & & & & $\begin{array}{l}\text { Fase 5: } \\
\text { Etapa }\end{array}$ \\
\hline cognitiva & & & & criativa \\
\hline $\begin{array}{l}\text { Fase 6: } \\
\text { Déficits }\end{array}$ & $\begin{array}{c}\text { Fase 13: } \\
\text { Diagnóstico } \\
\text { ergonômico }\end{array}$ & & & $\begin{array}{c}\text { Fase 6: } \\
\text { Geração de } \\
\text { alternativas } \\
\end{array}$ \\
\hline \multirow[t]{3}{*}{$\begin{array}{c}\text { Fase 7: } \\
\text { Potenciais }\end{array}$} & \multirow[t]{3}{*}{$\begin{array}{c}\text { Fase 14: } \\
\text { Recomendações } \\
\text { ergonômicas }\end{array}$} & & & $\begin{array}{c}\text { Fase 7: } \\
\text { Avaliação das } \\
\text { alternativas }\end{array}$ \\
\hline & & & & $\begin{array}{c}\text { Fase 8: } \\
\text { Seleção e } \\
\text { refinamento } \\
\text { da alternativa } \\
\end{array}$ \\
\hline & & & & $\begin{array}{l}\text { Fase 9: } \\
\text { Projeto } \\
\text { ergonômico }\end{array}$ \\
\hline
\end{tabular}

Figura 2. Fluxograma proposto: parte 1.

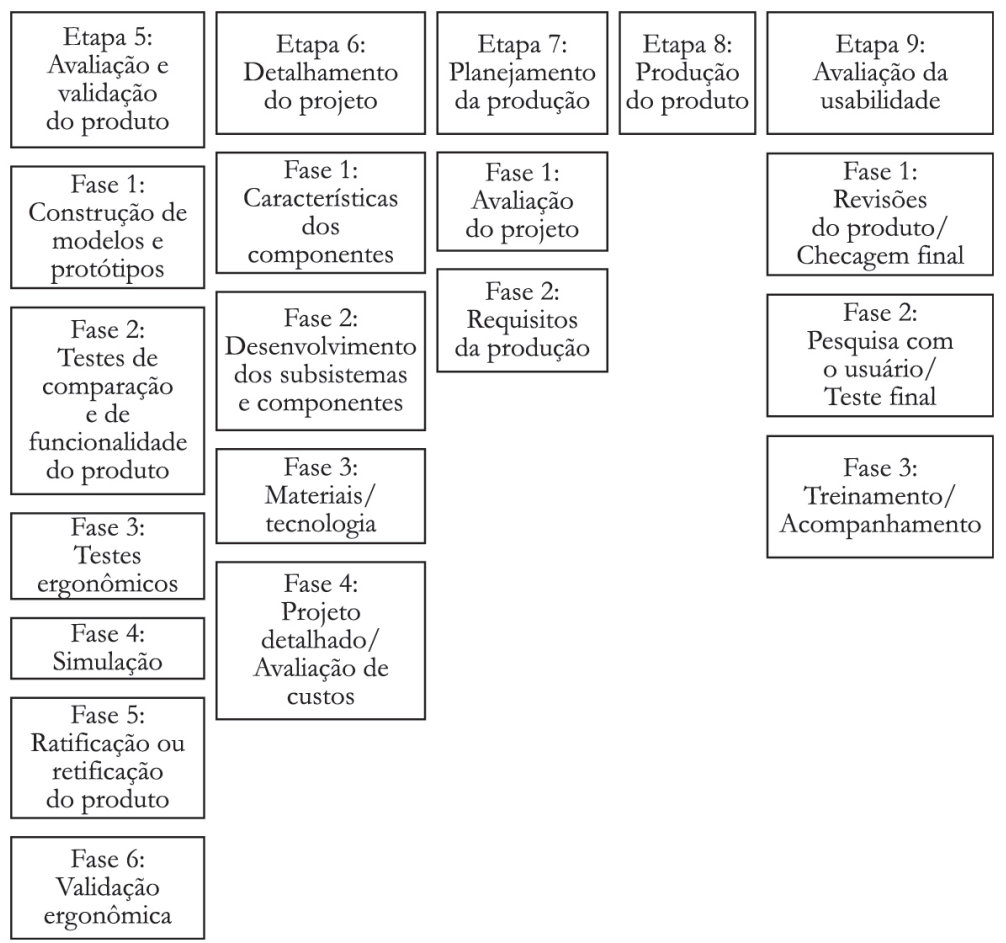

Figura 3. Fluxograma proposto: parte 2. 
Observa-se que o fluxograma conta com nove etapas. Cada uma apresenta um conjunto de atividades e técnicas de trabalho. A fase Projetação depende da conclusão das três fases anteriores. $\mathrm{O}$ ciclo de projetação é considerado completo somente após a avaliação final da usabilidade do produto desenvolvido.

É preciso ressaltar a importância de que o usuário participe de todas as etapas do desenvolvimento do produto. A partir da observação da relação usuário/ produto/meio e do levantamento das suas necessidades e desejos, assim como das suas opinióes e sugestóes durante todo o processo, é possível o desenvolvimento de produtos que atendam mais consistentemente a demanda dos usuários.

\section{Considerações finais}

A necessidade de se discutirem questóes relativas a produtos de TA é latente. O Brasil vem trilhando um caminho de busca e interesse em desenvolver e aprimorar os estudos e recursos nessa área.

Áreas como Ergonomia, Design e Engenharia vêm desenvolvendo um conjunto de métodos e técnicas que certamente poderão ser adaptados ao campo da Terapia Ocupacional, resultando em produtos mais bem desenvolvidos, capazes de atender tanto aos usuários quanto aos produtores. No campo do design, especificamente, a metodologia design centrado no usuário tem sido testada e resultados relevantes têm sido apresentados em congressos científicos da área.

\section{Referências}

ALVES, A. C. J. Tecnologia assistiva: identificação de modelos e proposição de um método de Implementação de recursos. 2013. 145 f. Tese (Doutorado em Educação Especial)-Universidade Federal de São Carlos, São Carlos, 2013.

BERSCH, R. C. R. Design de um serviço de tecnologia assistiva em escolas públicas. 2009. 231 f. Dissertação (Mestrado em Design)-Universidade Federal do Rio Grande do Sul, Porto Alegre, 2009.

CIPINIUK, A.; PORTINARI, D. Sobre métodos de design. In: COELHO, L. A. (Org.). Design método. Rio de Janeiro: Novas Idéias, 2006. p. 17-38.

DAY, H.; JUTAI, J. Measuring the psychosocial compact of assistive devices: the PIADS. Canadian Journal of Rehabilitation, Edmonton, v. 9, n. 2, p. 159-168, 1996.

DESMET, P.; DIJKHUIS, E. A wheelchair can be fun: a case of emotion-driven design. In: INTERNATIONAL CONFERENCE ON DESIGNING PLEASURABLE PRODUCTS AND INTERFACE - DPPI, 2003, Pittsburgh. Proceedings... New York: ACM, 2003. p. 22-27. Disponível em: <http://portal.acm.org/citation. cfm?id=782903>. Acesso em: 16 maio 2009.

FRISONI, B. C. Ergodesign, metodologia ergonômica, "designing" para o uso humano. 2000. 340 f. Dissertação (Mestrado em Design)-Pontifícia Universidade Católica do Rio de Janeiro, Rio de Janeiro, 2000.

HOCKING, C. Function or feelings: factors in abandonment of assistive devices. Technology and Disability, Amsterdam, v. 11, n. 1-2, p. 3-11, 1999.

JUTAI, J. Quality of life impact of assistive technology. Rehabilitation Engineering, Canada, v. 14, p. 2-7, 1999.

LARICA, N. Formigueiros virtuais. In: COELHO, L. A. (Org.). Design método. Rio de Janeiro: Novas Idéias, 2006. p. 87-98.

LOUISE-BENDER, P. T.; KIM, J.; WEINER, B. The shaping of individual meanings assigned to assistive technology: a review of personal factors. Disability and Rehabilitation, London, v. 24, n. 1-3, p. 5-20, 2002. http:// dx.doi.org/10.1080/09638280110066235

LUPTON, D.; SEYMOUR, W. Technology, selfhood and physical disability. Social Science \& Medicine, Oxford, v. 50, n. 12, p. 1851-1862, 2000. http://dx.doi.org/10.1016/ S0277-9536(99)00422-0

MALLIN, S. S. Uma metodologia de Design aplicada ao desenvolvimento de tecnologia assistiva para portadores de paralisia cerebral. Curitiba: UFPR, 2004.

MANZINI, E.; SANTOS, M. C. Recursos pedagógicos adaptados: equipamento e material pedagógico especial para educação, capacitação e recreação da pessoa com deficiência física. Brasília: Ministério de Educação: Secretaria de Educação Especial, 2002.

PHILLIPS, B.; ZHAO, H. Predictors of assistive technology abandonment. Assistive Technology: The Official Journal of RESNA, New York, v. 5, n. 1, p. 36-45, 1993. POULSON, D.; ASHBY, M.; RICHARDSON, S. J. (Ed.). USERFIT: a practical handbook on user-centred design for assistive technology. Luxemburgo: HUSAT Research Institute for the European Commission, 1996. Disponível em: <http://ec.europa.eu/information_society/ activities/einclusion/archive/deploy/dfa/index_en.htm>. Acesso em: 28 fev. 2010.

RIEMER-REISS, M. L.; WACKER, R. R. Factors associated with assistive technology discontinuance among individuals with disabilities. The Journal of Rehabilitation, Alexandria, v. 66, n. 3, p. 44-50, 2000.

ROCHA, A. N. D. C. Processo de prescriçãa e confecção de recursos de tecnologia assistiva para educação infantil. 2010. 201 f. Dissertaçáo (Mestrado em Educação)-Universidade Estadual Paulista, Marília, 2010.

SCHERER, M. J. et al. Predictors of assistive technology use: the importance of personal and psychosocial factors. Disability and Rehabilitation, London, v. 27, n. 21, p. 1321-1331, 2005. PMid:16298935. http://dx.doi. org/10.1080/09638280500164800

SCHERER, M. J. The change in emphasis from people to person: introduction to the special issue on 
assistive technology. Disability and Rehabilitation, London, v. 24, n. 1-3, p. 1-4, 2002. http://dx.doi. org/10.1080/09638280110066262

SCHERER, M.; CRADDOCK, G. Matching Person and Technology (MPT): assessment process. Technology and Disability, Amsterdam, v. 14, n. 3, p. 125-131, 2002.

SOUZA, H. L. GRAFCET como ferramenta no desenvolvimento de tecnologia assistiva. 2004. $132 \mathrm{f}$. Dissertação (Mestrado em Ciências Básicas)-Universidade de São Paulo, São Carlos, 2004.
WEISS-LAMBROU, R. et al. Weelchair seat aids: how satisfied are consumers? Assistive Technology: The Official Journal of RESNA, New York, v. 11, n. 1, p. 43-53, 2000. WU, F. G.; MA, M. Y.; CHANG, R. H. A new usercentered design approach: a hair washing assistive device design for users with shoulder mobility restriction. Applied Ergonomics, London, v. 40, n. 5, p. 878-886, 2009. PMid:19232409. http://dx.doi.org/10.1016/j. apergo.2009.01.002

\section{Contribuição dos Autores}

Fernanda do Nascimento Maia: concepção do texto manuscrito. Sydney Fernandes de Freitas: orientação e revisão do texto. Ambos os autores aprovaram a versão final. 